7th International Workshop on Astronomy and

Relativistic Astrophysics (IWARA 2016)

International Journal of Modern Physics: Conference Series

Vol. 45 (2017) 1760044 (8 pages)

(C) The Author(s)

DOI: $10.1142 / S 2010194517600448$

\title{
Chiral Symmetry Restoration for Quark Matter with a Chiral Chemical Potential
}

\author{
Ricardo L. S. Farias, Dyana C. Duarte \\ Departamento de Física \\ Universidade Federal de Santa Maria (UFSM) \\ 97105-900 Santa Maria, RS, Brazil \\ ricardo.farias@ufsm.br,dyana.c.duarte@gmail.com \\ Gastão Krein and Rudnei O. Ramos \\ Instituto de Física Teórica \\ Universidade Estadual Paulista (UNESP) \\ 01049-010 São Paulo, SP, Brazil \\ gkrein@ift.unesp.br,rudnei@uerj.br
}

Published 15 August 2017

\begin{abstract}
In this work we study the chiral transition in quark matter at finite temperature and in the presence of a chiral imbalance in the Nambu-Jona-Lasinio model. We use the Medium Separation Scheme (MSS) to rewrite divergent momentum integrals that appear in the model in such way that we can conciliate results obtained with the model and recent lattice results for the chiral transition.
\end{abstract}

Keywords: Chiral Imbalance, Chiral Transition, Medium Separation.

PACS numbers: 12.38.Mh, 21.65.Qr, 12.39.Ki

\section{Introduction}

The fact that a chiral imbalance of right- and left-handed quarks in quark matter can influence the phase diagram of quantum chromodynamics (QCD) has motivated many investigations recently. There are different reasons for this interest, e.g the nontrivial nature of the vacuum of QCD that allows for the existence of topological field configurations like instantons and sphalerons; the latter, in particular, can generate an asymmetry between the number of left- and right-handed quarks due to the Adler-Bell-Jackiw anomaly. This chiral imbalance in quark matter is expected to occur in event-by-event $C$ - and $C P$-violating processes in heavy-ion collisions ${ }^{1,2}$.

This is an Open Access article published by World Scientific Publishing Company. It is distributed under the terms of the Creative Commons Attribution 4.0 (CC-BY) License. Further distribution of this work is permitted, provided the original work is properly cited. 


\section{R. L. S. Farias et al.}

Another interesting scenario where a chiral imbalance plays an important role is in the chiral magnetic effect (CME): in this case, a charge separation can be produced due the magnetic field created in off-central collisions that, in the presence of a chiral imbalance, gives rise to an electric current along the magnetic field-Ref. ${ }^{3}$ provides a review with is a large list of references. The CME can be found in different physical systems and actually has been observed experimentally in semi-metalsfor a recent review, see Ref. ${ }^{4}$.

Effects of a chiral imbalance on the QCD phase diagram can be studied in the grand canonical ensemble introducing a chiral chemical potential $\mu_{5}$ via a term $\mu_{5} \bar{\psi} \gamma_{0} \gamma_{5} \psi$ in the QCD Lagrangian ${ }^{2}$. This allows, at least in principle, mapping the QCD phase diagram in the $T-\mu_{5}$ plane into the real phase diagram in the $T-\mu$ plane ${ }^{5,6}$, where $\mu$ is the baryon chemical potential. That is, such a mapping can be helpful in finding the expected critical end point (CEP) of the chiral transition (see Refs. ${ }^{7,8}$ for opposite views). An additional interesting feature of QCD at finite $\mu_{5}$ is that that it is free from the sign problem that afflicts QCD at finite $\mu$ and, therefore, it is amenable to Monte Carlo sampling in lattice simulations.

Recent lattice QCD results ${ }^{9,10}$ at finite $T$ and $\mu_{5}$ are, to some extend surprisingly, in contradiction with all corresponding results obtained with effective models of QCD. Results based on Nambu-Jona-Lasinio (NJL)-type of models ${ }^{5,11-15}$ and quark linear sigma models ${ }^{5,16}$ predict that $T_{c}$ decreases with $\mu_{5}$, while the lattice results of Refs. ${ }^{9,10}$ find the opposite behavior. The contradiction came as a surprise since the models have been very helpful in giving insight in different pieces of chiral physics and seem to fail in this particular chiral problem. On the other hand, the behavior found for $T_{c}$ in lattice simulations seems to be expected on general physical grounds, as the addition of left- and right-handed quarks to a system favors quark-antiquark pairing, that is, $\mu_{5}$ increases the quark condensate ${ }^{17}$. Universality arguments for QCD in the limit of a large number of colors $N_{c}{ }^{18}$ lend further support to the lattice results. Different approaches have appeared in the recent literature trying to understand this discrepancy, e.g. studies based using phenomenological quark-gluon interactions in the framework of the Dyson-Schwinger equations (DSE) for the quark propagator ${ }^{7,8}$ and nonlocal finite-range NJL models ${ }^{19,20}$ find a $T_{c}$ increasing with $\mu_{5}$. A qualitative agreement with the lattice results for $T_{c}$ was also found in Ref. ${ }^{21}$ using the quark linear sigma model with a nonstandard renormalization scheme.

The motivation for this work is try to understand why the local version of the NJL model do not reproduce the behavior for $T_{c}$ as a function of $\mu_{5}$ founded in lattice simulations. In a recent work ${ }^{22}$, we propose that this discrepancy can be solved using a strategy based in a proper separation of the medium effects from the divergent integrals (Medium Separation scheme (MSS)), so that all divergent integrals are the same as those that apear in the vacuum $\left(T=0\right.$ and $\left.\mu_{5}=0\right)$. The improper separation of the medium effects can introduce unphysical effects ${ }^{23-25}$. Our procedure consist in rewrite all divergent integras in terms of integrals that appear in the vacuum, once the divergent integrals are fixed to reproduce physical 
results, our results predicts that $T_{c}$ is an increasing function of $\mu_{5}$. We aplly MSS in the NJL model, but it can be applied in other effective models for QCD like the Polyakov-Nambu-Jona-Lasinio (PNJL) model ${ }^{11,15}$.

This paper is organized as follows. In Sec. 2 we describe the model and the regularization scheme used to implement se separation of the medium effects. In Sec. 3 we compare our numerical results obtained in the context of this MSS with the traditional cutoff scheme (traditional regularization scheme(TRS)). Our final remarks and conclusions are presented in Sec. 4.

\section{Chiral Imbalanced NJL Model}

The version of the NJL model including chiral chemical potential is given by the Lagrangian density:

$$
\mathcal{L}=\bar{\psi}\left(i \not \partial-m_{c}+\mu_{5} \gamma^{0} \gamma^{5}\right) \psi+G\left[(\bar{\psi} \psi)^{2}+\left(\bar{\psi} i \gamma_{5} \vec{\tau} \psi\right)^{2}\right]
$$

where $m_{c}$ is the current quark mass $\left(m_{c}=0\right.$ in the quiral limit), $G$ is the coupling and $\psi$ represents a quark field. There are implicit sums over flavors, $N_{f}=2$ and over color, $N_{c}=3$. The thermodynamic potential of the model in mean field approximation can be written as

$$
\begin{aligned}
\Omega\left(M, T, \mu_{5}\right)= & \frac{\left(M-m_{c}\right)^{2}}{4 G}-N_{f} N_{c} \sum_{s= \pm 1} \int \frac{d^{3} k}{(2 \pi)^{3}} \omega_{s}(k) \\
& -2 N_{f} N_{c} T \sum_{s= \pm 1} \int \frac{d^{3} k}{(2 \pi)^{3}} \ln \left[1+e^{-\omega_{s}(k) / T}\right],
\end{aligned}
$$

where the dynamical quark mass $M \equiv M\left(T, \mu_{5}\right)$ is given by the gap equation

$$
\partial \Omega\left(M, T, \mu_{5}\right) / \partial M=0
$$

and

$$
\omega_{s}(k)=\sqrt{\left(|\mathbf{k}|+s \mu_{5}\right)^{2}+M^{2}} .
$$

The momentum integral in the first line of Eq. (2) is UV divergent and requires regularization.

The MSS scheme of separating medium-dependent quantities from the integrands of divergent integrals proceeds as follows. Initially, we note that in the gap equation, which comes from the derivation of $\Omega\left(M, T, \mu_{5}\right)$ with respect to $M$, there appears a quadratic divergence

$$
\frac{\partial}{\partial M^{2}}\left[\int \frac{d^{3} k}{(2 \pi)^{3}} \omega_{s}(k)\right]=\int_{-\infty}^{+\infty} \frac{d k_{4}}{2 \pi} \int \frac{d^{3} k}{(2 \pi)^{3}} \frac{1}{k_{4}^{2}+\omega_{s}^{2}(k)} .
$$

Next, the integrand of the last integral is rewritten using identity 26,23

$$
\begin{aligned}
\frac{1}{k_{4}^{2}+\omega_{s}^{2}(k)} & =\frac{1}{k_{4}^{2}+k^{2}+M_{0}^{2}}-\frac{A_{s}(k)}{\left(k_{4}^{2}+k^{2}+M_{0}^{2}\right)^{2}}+\frac{A_{s}^{2}(k)}{\left(k_{4}^{2}+k^{2}+M_{0}^{2}\right)^{3}} \\
& -\frac{A_{s}^{3}(k)}{\left(k_{4}^{2}+k^{2}+M_{0}^{2}\right)^{3}\left[k_{4}^{2}+\omega_{s}^{2}(k)\right]}
\end{aligned}
$$




\section{R. L. S. Farias et al.}

where

$$
A_{s}(k)=\mu_{5}^{2}+2 s k \mu_{5}+M^{2}-M_{0}^{2},
$$

and $M_{0}$ is the quark mass in the vacuum, solution of the gap equation at $T=0$ and $\mu_{5}=0$. Finally, the gap equation can then be written as

$$
\begin{aligned}
\frac{M-m_{c}}{4 N_{f} N_{c} G M}= & I_{\text {quad }}\left(\Lambda, M_{0}\right)+\left(2 \mu_{5}^{2}-M^{2}+M_{0}^{2}\right) I_{\log }\left(\Lambda, M_{0}\right)-\frac{2 \mu_{5}^{2}+M^{2}-M_{0}^{2}}{8 \pi^{2}} \\
& +\frac{M^{2}-2 \mu_{5}^{2}}{8 \pi^{2}} \ln \left(\frac{M^{2}}{M_{0}^{2}}\right)-\sum_{s= \pm 1} \int \frac{d^{3} k}{(2 \pi)^{3}} \frac{1}{\omega_{s}(k)} \frac{1}{e^{\omega_{s}(k) / T}+1},
\end{aligned}
$$

where

$$
I_{\text {quad }}\left(\Lambda, M_{0}\right)=\int^{\Lambda} \frac{d^{4} k}{(2 \pi)^{4}} \frac{1}{k_{4}^{2}+k^{2}+M_{0}^{2}}, \quad I_{\log }\left(\Lambda, M_{0}\right)=-I_{\text {quad }}^{\prime}\left(\Lambda, M_{0}\right),
$$

the prime in $I_{\text {quad }}^{\prime}$ means derivative with respect to $M_{0}^{2}$. Note that where $M_{0}$ is the vacuum quark mass and $\Lambda$ denotes the regularization parameter used in the divergent integrals. The values of $I_{\text {quad }}$ and $I_{\log }$ are fixed by fitting vacuum properties: quark condensate $\langle\bar{q} q\rangle$, the leptonic decay constant $f_{\pi}$ and the pion mass $m_{\pi}$. We also note to arrive to the result in Eq. (5), all finite integrals are integrated without a regulator; the same is done with the last integral in this equation that depends explicitly in temperature and $\mu_{5}$.

The main point here is that the divergent integrals do not present explicit or implicit dependence on $T$ or $\mu_{5}$ in their integrands, they are the same that appear in the vacuum of the model. We can reguralize the divergent integrals with different schemes, as, e.g., by a three-dimensional momentum cutoff $\Lambda$, and fix $\Lambda$ by fitting a vacuum physical quantity.

In addition, other works that have used TRS in different effective models of QCD ${ }^{5,6,11-14,16}$ they also find a CEP on the phase diagram $\left(\mu_{5}, T_{c}\right)$. The recent lattice results ${ }^{9,10}$ show explicitly that besides $T_{c}$ increasing with $\mu_{5}$ they also obtain a transition that is only a crossover.

\section{Numerical Results}

Our results for the dynamical quark mass $M$ as a function of $\mu_{5}$ at $T=0$ are presented in Fig. 1. We can see that TRS scheme seems to indicate that the chiral chemical potential initially strengthens dynamical chiral symmetry breaking (DCSB), but this behavior changes at around $\mu_{5} \simeq 0.6 \Lambda$, beyond which it starts to disfavor DCSB. However, in the MSS scheme, DCSB is always strengthened by the chiral chemical potential. This change of behavior is due the separation of the medium effects from vacuum effects. This separation will also have an impact on how the critical temperature changes with $\mu_{5}$.

We use a three-dimensional cutoff to evaluate the vacuum divergent integrals. The values of the parameters are: $m_{c}=5.37 \mathrm{MeV}, G=4.75 \mathrm{GeV}^{-2}$ and $\Lambda=$ 


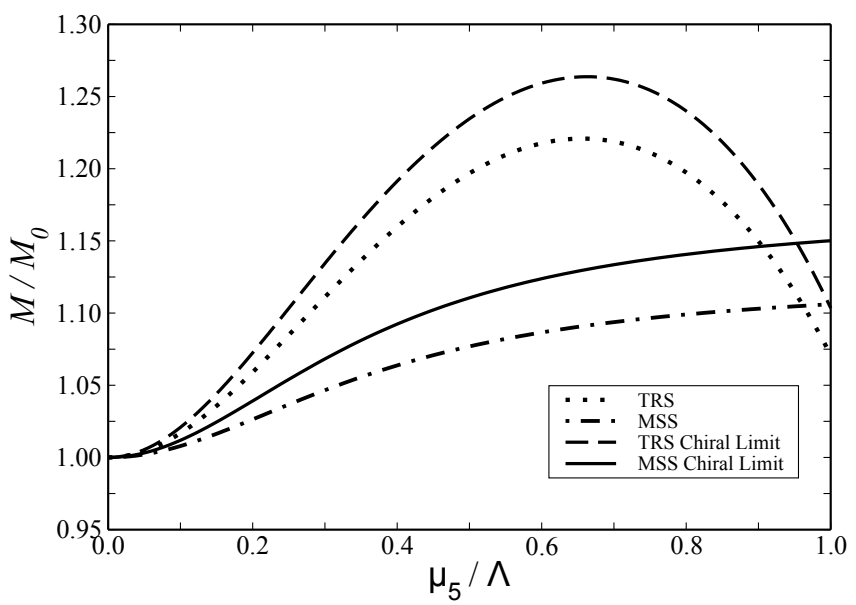

Fig. 1. The zero temperature quark mass $M$, normalized by its respective vacuum value $M_{0}$, as a function of chiral chemical potential $\mu_{5}$. The results obtained using the TRS regularization are given by the dashed (chiral limit) and dotted lines. In the MSS regularization the results are given by the solid (chiral limit, $m_{c}=0$ ) and dash-dotted lines.

$0.660 \mathrm{GeV}$; they are fixed using as input $\langle\bar{q} q\rangle^{1 / 3}=-0.250 \mathrm{GeV}, m_{\pi}=0.140 \mathrm{GeV}$ and $f_{\pi}=92.3 \mathrm{MeV}$. The constituent quark mass in the vacuum is $M_{0}=0.302 \mathrm{GeV}$.

Table 1. Critical $T_{c}$ and pseudo-critical $T_{p c}$ temperatures at $\mu_{5}=0$.

\begin{tabular}{ccc}
\hline Reg. scheme & $T_{c}[\mathrm{GeV}]$ & $T_{p c}[\mathrm{GeV}]$ \\
\hline TRS & 0.165 & 0.177 \\
MSS & 0.169 & 0.183 \\
\hline
\end{tabular}

In Fig. 2 we show the results for the critical temperature $T_{c}$ as a function of $\mu_{5}$ using TRS and MSS. The values of $T_{0}$, for the critical $\left(T_{c}\right)$ and pseudo-critical $\left(T_{p c}\right)$ temperatures for chiral symmetry restoration evaluated at $\mu_{5}=0$ are given in Tab. 1.

In the results of TRS scheme there appears a critical end point (CEP) that separates a crossover line from a first-order transition. In the chiral limit $\left(m_{c}=0\right)$, the transition is instead a tri-critical point (TP), which separates a line of secondorder phase transition from one of first-order. In the MSS scheme, the transition is a crossover and in the chiral limit, the transition is second order throughout. Other very important result is that in MSS scheme one sees that $T_{c}$ always increases with $\mu_{5}$. The MSS results are in qualitative accordance with the recent results from the lattice $^{9,10}$ and also with other non-perturbative treatments ${ }^{7,8}$. The absence of the 


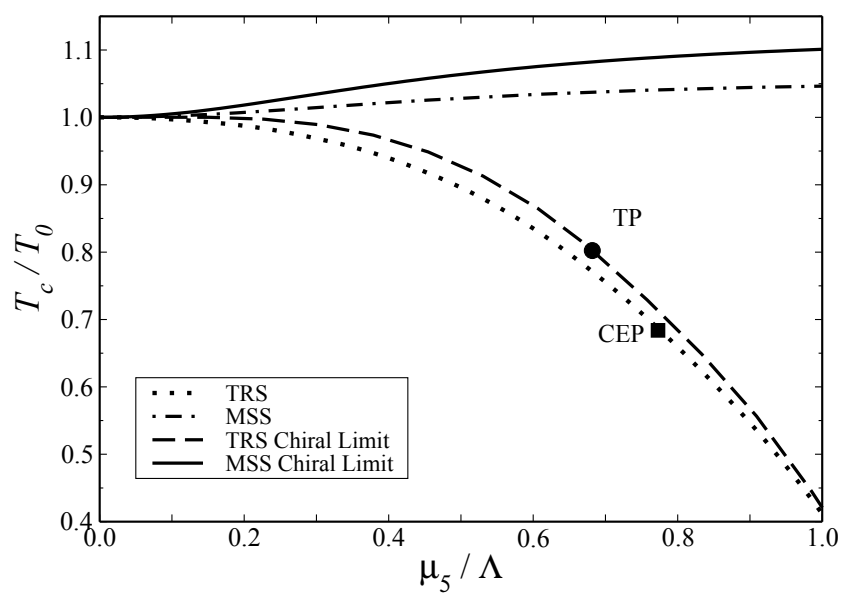

Fig. 2. The critical temperature $T_{c}$, normalized by $\left.T_{0}=T_{c}\left(\mu_{5}=0\right)\right)$, as a function of $\mu_{5}$. The black dot indicates a tricritical point in the chiral limit, while the square indicates the critical end point, both in the TRS regularization case (see text for a detailed explanation).

TP (in the chiral limit) or the CEP in the MSS regularization is also seen in the results obtained from the earlier lattice results ${ }^{27}$, and also with the more recent ones, where no CEP (or TP) has been found. One should mention that the lattice results in Refs. ${ }^{9,10}$ should be taken with some caution, as they were obtained for a very large pion mass, $m_{\pi}=418 \mathrm{MeV}$.

\section{Conclusions}

We have shown a way to conciliate results obtained with the NJL model and recent lattice results for the critical temperature of chirally imbalanced quark matter. We pointed out that the discrepancy between the model and lattice results is strongly connected on how the UV momentum integrals are treated in these NJL-type of models.

Using the MSS, we implement a proper separation of medium effects from vacuum effects from the integrand of the divergent integrals that require regularization; finite integrals are performed without any cutoff, in the same way as the integrations over the terms depending explicitly on $T$ are performed. Our results for the critical temperature dependence with the chiral chemical potential are in qualitative agreement with physical expectations, in that $\mu_{5}$ is a catalyst of DCSB ${ }^{17}$ and, therefore, an increasing critical temperature as a function of $\mu_{5}$ should be expected. Another result that we also have is a qualitative agreement with lattice results regarding the absence of a CEP. It it important to mention that recent studies ${ }^{7,8}$ based on a renormalizable, non-perturbative scheme based on the DSE of QCD also do not find a critical end point in the $\left(T, \mu_{5}\right)$ phase diagram - see also discussions 
of Zhu-Fang Cui et al. ${ }^{15}$. It would be important to examine such result within similar approaches using DSE in QCD, particularly in models that incorporate different confining mechanisms, like those inspired in Coulomb gauge ${ }^{28}$ and soliton models ${ }^{29}$. The regularization scheme used in this work can be relevant for other problems that perform a mixing of medium and vacuum terms. We believe that our approach can improve the predictability of these effective models of QCD, which can help to study the QCD phase diagram.

\section{Acknowledgments}

Work partially financed by Conselho Nacional de Desenvolvimento Científico e Tecnológico - CNPq, Grant Nos. 305894/2009-9 (G.K.), 475110/2013-7 (R.L.S.F), 232766/2014-2 (R.L.S.F), 308828/2013-5 (R.L.S.F) and 303377/2013-5 (R.O.R.), Fundação de Amparo à Pesquisa do Estado de São Paulo - FAPESP, Grant No. 2013/01907-0, Fundação Carlos Chagas Filho de Amparo à Pesquisa do Estado do Rio de Janeiro (FAPERJ), Grant No. E - 26 / 201.424/2014 (R.O.R.) and CAPES (D.C.D). R. L. S. F. acknowledges the kind hospitality of the Center for Nuclear Research at Kent State University, where part of this work has been done. R. L. S. F. is also grateful to Michael Strickland for insightful comments and suggestions. We thank A. Y. Kotov for comments and discussions.

\section{References}

1. D. Kharzeev, Phys. Lett. B 633, 260 (2006).

2. K. Fukushima, D. E. Kharzeev, and H. J. Warringa, Phys. Rev. D 78, 074033 (2008).

3. D. E. Kharzeev et al., Prog. Part. Nucl. Phys. 88, 1 (2016).

4. Q. Li and D. E. Kharzeev, Nucl. Phys. A 956, 107 (2016).

5. M. Ruggieri, Phys. Rev. D 84, 014011 (2011).

6. R. Gatto and M. Ruggieri, Phys. Rev. D 85, 054013 (2012).

7. B. Wang, Y. L. Wang, Z. F. Cui, and H. S. Zong, Phys. Rev. D 91, 034017 (2015).

8. S. S. Xu et al. Phys. Rev. D 91, 056003 (2015).

9. V. V. Braguta et. al. Phys. Rev. D 93, 034509 (2016).

10. V. V. Braguta et al., JHEP 1506, 094 (2015)

11. K. Fukushima, M. Ruggieri, and R. Gatto, Phys. Rev. D 81, 114031 (2010).

12. J. Chao, P. Chu, and M. Huang, Phys. Rev. D 88, 054009 (2013).

13. L. Yu, H. Liu, and M. Huang, Phys. Rev. D 90, 074009 (2014)

14. L. Yu, H. Liu, and M. Huang, Phys. Rev. D 94 no.1, 014026 (2016).

15. Zhu-Fang Cui et al., Phys. Rev. D 94, 071503 (2016).

16. M. N. Chernodub and A. S. Nedelin, Phys. Rev. D 83, 105008 (2011).

17. V. V. Braguta and A. Yu. Kotov, Phys. Rev. D 93, 105025 (2016).

18. M. Hanada and N. Yamamoto, PoS LATTICE 2011, 221 (2011).

19. M. Frasca, arXiv:1602.04654 [hep-ph](2016).

20. M. Ruggieri and G. X. Peng, arXiv:1602.05250 [hep-ph](2016).

21. M. Ruggieri and G. X. Peng, arXiv:1602.03651 [hep-ph](2016).

22. R. L .S. Farias, D.C. Duarte, G. Krein, and R. O. Ramos, Phys. Rev. D 94, 074011 (2016).

23. R. L. S. Farias, G. Dallabona, G. Krein, and O. A. Battistel, Phys. Rev. C 73, 018201 (2006). 
24. P. G. Allen, A. G. Grunfeld, and N. N. Scoccola, Phys. Rev. D 92, 074041 (2015).

25. D. C. Duarte, P. G. Allen, R. L. S. Farias, P. H. A. Manso, R. O. Ramos, and N. N. Scoccola, Phys. Rev. D 93, 025017 (2016).

26. O. A. Battistel and M. C. Nemes, Phys. Rev. D 59, 055010 (1999).

27. A. Yamamoto, Phys. Rev. Lett. 107, 031601 (2011).

28. P. J. A. Bicudo et al., Phys. Rev. D 45, 1673 (1992).

29. G. Krein et al., Phys. Lett. B 212, 362 (1988). 